\title{
Retinal ganglion cell death is triggered by paraptosis via reactive oxygen species production: A brief literature review presenting a novel hypothesis in glaucoma pathology
}

\author{
YAO WANG ${ }^{1,2}, \mathrm{KUN} \mathrm{XU}^{2}, \mathrm{HONGBING} \mathrm{ZHANG}^{2}, \mathrm{JUNHONG} \mathrm{ZHAO}^{2}$, \\ XIUPING ZHU ${ }^{2}$, YANGZHENG WANG ${ }^{2}$ and RENYI WU ${ }^{1}$ \\ ${ }^{1}$ Eye Institute and Affiliated Xiamen Eye Center, Xiamen University, Fujian Provincial Key Laboratory of \\ Ophthalmology and Visual Science, Xiamen, Fujian 361102; ${ }^{2}$ Department of Ophthalmology, First Hospital of Xi'an, \\ Shaanxi Institute of Ophthalmology, Shaanxi Provincial Key Laboratory of Ophthalmology, \\ Xi'an, Shaanxi 710002, P.R. China
}

Received August 21, 2013; Accepted May 13, 2014

DOI: $10.3892 / \mathrm{mmr} .2014 .2346$

\begin{abstract}
Accumulative evidence has indicated that apoptosis is the common pathway for retinal ganglion cell (RGC) death and that autophagy promotes survival of RGCs in glaucoma. In the present review, it was hypothesized that the progressive death of RGCs in glaucoma involves another novel non-apoptotic programmed cell death, known as 'paraptosis', in the early stages of glaucoma. Paraptosis may be accompanied by apoptosis and/or autophagy in the moderate and severe stages. The secondary hypothesis suggests that paraptosis in glaucomatous RGCs may be triggered by damage to cellular mitochondria, and is associated with mitochondria-derived reactive oxygen species (ROS). Our preliminary laboratory studies, using transmission electron microscopy, provided evidence that supports the primary hypothesis. The secondary hypothesis is currently under investigation. These two hypotheses provide a novel way to investigate the mechanisms of cell death in glaucomatous RGCs and targeting paraptosis may be a promising strategy for RGC-protecting drug discovery.
\end{abstract}

Correspondence to: Professor Renyi Wu, Eye Institute and Affiliated Xiamen Eye Center, Xiamen University, Xiang-an Road, Xiamen, Fujian 361102, P.R. China

E-mail:wubasel@hotmail.com

Dr Yao Wang, Department of Ophthalmology, First Hospital of Xi'an, Shaanxi Institute of Ophthalmology, No. 30 Fen Xiang, South Street, Xi'an, Shaanxi 710002, P.R. China

E-mail:wangyao0605@yeah.net

Key words: retinal ganglion cell death, glaucoma, paraptosis, mitochondria, reactive oxygen species

\section{Contents}

1. Introduction

2. Hypothesis

3. Evidence of hypothesis

4. Preliminary findings of hypothesis

5. Conclusion

\section{Introduction}

Glaucoma is a serious and irreversible disease that causes blindness and is commonly termed the 'silent thief of sight', because loss of vision often occurs gradually. In the majority of cases, glaucoma is associated with intraocular pressure and low blood perfusion, and is characterized by progressive death of retinal ganglion cells (RGCs). As glaucoma progresses, it may lead to optic nerve degeneration and loss of retinal nerve fibers, and ultimately result in the loss of vision. So, facilitating the survival of RGCs may be an effective therapeutic target in the treatment of glaucoma. Within the retina, RGCs are the only projecting neurons that transmit visual information to the brain (1), representing the third-order neurons of the visual pathway (2). Following optic nerve injury, RGCs are unable to regenerate their axons and cell apoptosis is rapidly initiated (3).

Numerous studies have indicated that apoptosis may be the final common pathway for RGC death in glaucoma (4-7). However, it has become increasingly evident that apoptosis and necrosis alone do not adequately encompass cell death. Studies have revealed that although apoptosis is actively executed by cysteine-aspartic acid proteases (caspase), RGC death occurs independently of caspase-mediated pathways $(8,9)$, which are a family of cysteine proteases that are also essential in necrosis and inflammation (10). These data imply that RGC death involves apoptosis as well as non-apoptotic programmed cell death (PCD). Autophagy is a type of cell death that is morphologically distinct from apoptosis and which usually mediates cytoprotection, avoiding the apoptotic or necrotic demise of cells $(11,12)$. Activation of autophagy reduces 
or inhibits the apoptosis of the cells. Several studies have provided evidence that the survival of RGCs may be improved by autophagy following optic nerve axotomy in mice $(13,14)$. These investigations demonstrated that besides from apoptosis and necrosis, other pathways may also be responsible for RGC death. The accumulating literature providing evidence of the mechanisms of non-apoptotic RGC death suggests that other possible pathways are involved.

Cell death is categorized into PCD and passive (necrotic) cell death $(15,16)$. While apoptosis is the best characterized form of PCD, other non-apoptotic modes also exist, including autophagy, paraptosis and mitotic catastrophe (16). Autophagy, as a form of PCD, is a self-degradative process that involves the breakdown of intracellular proteins and organelles (17). Paraptosis is a novel type of PCD, that is caspase independent and is characterized by cytoplasmic vacuolization derived from the endoplasmic reticulum and/or mitochondria swelling. Paraptosis is distinguished from apoptosis by its non-response to caspase inhibitors (that block apoptosis) and lack of apoptotic morphology. This type of cell death is also insensitive to autophagic inhibitors and lacks DNA fragment and poly (ADP-ribose) polymerase (PARP) cleavage $(15,18)$. Several studies have identified AIP-1/Alix and phosphatidylethanolamine binding protein 1 (PEBP-1) as inhibitors of paraptosis $(15,19)$.

Paraptosis is fundamentally different from other forms of PCD, such as apoptosis, autophagy and oncosis. Apoptosis typically involves the activation of caspases, responds to caspase inhibitors and exhibits morphological features including chromatin condensation, nuclear fragmentation and the formation of apoptotic bodies. Autophagy exhibits a contrasting morphology during cytoplasmic vacuolization in comparison with paraptosis. The process of autophagy is the formation of autophagosomes, which are double-membrane vesicles responsible for delivering long-lived proteins and excess or damaged organelles, into the lysosome for degradation (20). In addition, paraptosis is not inhibited by autophagy inhibitors, including 3-mathyladenine (3-MA). Oncosis is another form of non-apoptotic PCD, which is distinguished from paraptosis and occurs in the destroyed cell membrane.

\section{Hypothesis}

In the present review, it was hypothesized that the progressive death of the RGCs in glaucoma is associated with a distinguished form of cell death, aside from the well characterized apoptosis, necrosis and autophagy, termed paraptosis (Fig. 1). Paraptosis may be triggered in the early stages of glaucoma (such as the appearance of optic nerve abnormalities, consistent with glaucoma, but with no visual field abnormalities). Furthermore, it was hypothesized that paraptosis and apoptosis may, together with autophagy, occur alone or simultaneously in RGCs, depending not only on the clinical severity but also on the duration of glaucoma development.

The second hypothesis is that paraptosis in glaucomatous RGCs may be derived from damage to the mitochondria. Mitochondria destruction plays a key role in inducing paraptosis in glaucomatous RGCs, and this may be associated with mitochondria-derived reactive oxygen species (ROS) reactions.



Figure 1. Proposed models of programmed RGC death pathways in glaucoma. The mechanisms by which autophagy antagonizes or enables apoptotic cell death and cross-talk between autophagy and apoptosis in RGCs has been illustrated. Paraptosis and apoptosis, even together with autophagy, may occur alone or simultaneously. Whether cross-talk exists between paraptosis and autophagy or apoptosis in RGCs remains to be elucidated. RGCs, retinal ganglion cells; ROS, reactive oxygen species.

\section{Evidence of hypothesis}

Paraptosis occurs in retinal pigment epithelial cells (RPEs) and retinal Müller glial cells (RMGs). Triamcinolone acetonide (TA), a synthetic corticosteroid, has been demonstrated to induce retinal toxicity via mechanisms predominantly associated with paraptosis in rat RPEs, RMGs and in ARPE-19 cells (human RPE cells) in vitro (21). However, whether TA induces paraptosis in RGCs has not been investigated. Nevertheless, these observations suggest that paraptosis may be triggered in retinal cells in certain conditions.

Glaucomatous RGCs are associated with the occurrence of paraptosis. Mechanisms of RGC death in glaucoma include neurotrophic factor deprivation, hypoperfusion/ischemia, glial cell activation, glutamate excitotoxicity and abnormal immune response (4). Paraptosis may be the mechanism of cell death in a number of pathological conditions, including excitotoxicity, ischemia and neurodegeneration (19), which suggests that the mechanisms of pathological damage in RGCs may be associated with the occurrence of paraptosis.

In addition, numerous studies have proved the importance of paraptosis in the nervous system. In one study, polymorphonuclear leukocytes and macrophages induced evident paraptosis in T9 glioma cells (22). Similarly, in human U251MG glioma cells expressing the membrane form of macrophage colony-stimulating factor (mM-CSF), cell death was induced by human monocytes in vitro and these cells were rejected within immunodeficient mice via the paraptosis pathway (23). Furthermore, intracellular acidification, by inhibition of the $\mathrm{Na}^{+} / \mathrm{H}^{+}$-exchanger, triggered caspase-independent cell death that resembles paraptosis in cerebellar granule neurons (24). As is well established, RGCs are a specialized type of neuron that are located near to the inner surface of the retina, which suggests that paraptosis may also have a role in the pathophysiological processes that lead to RGC damage. 

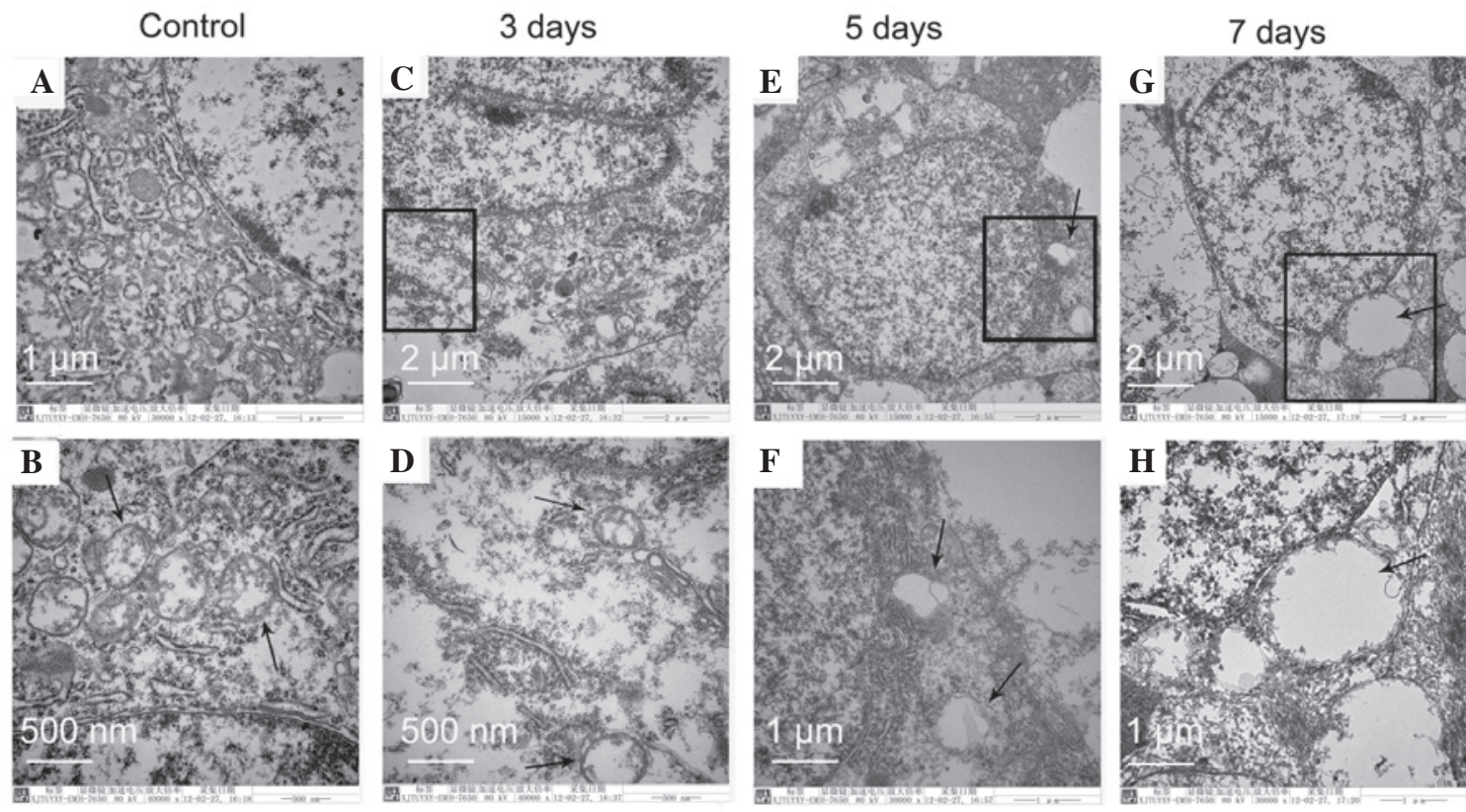

Figure 2. Electron microscopy analysis of RGCs, following optic nerve crush injury by clamping, indicates vacuoles have originated from the mitochondria. (A and B) Control cells with normal mitochondria, endoplasmic reticulum and uncondensed chromatin. (C and D) Swollen mitochondria with disintegrated cristae and decreased matrix density. (E-H) Images demonstrate that mega mitochondria are derived from swelling and fusion among mitochondria. Condensed chromatin and nuclear fragmentation are not detected. These morphologic features are consistent with the characteristics of the type of cell death termed paraptosis. (D, F and H) Enlarged images in the black squares of images (C, E and G), respectively.. The representative sections from three rats in each group are presented. The black arrows indicate the mitochondria. Original magnifications: $(\mathrm{C}),(\mathrm{E})$ and $(\mathrm{G}): \mathrm{x} 15,000 ;(\mathrm{A}),(\mathrm{F})$ and $(\mathrm{H}): \mathrm{x} 30,000 ;(\mathrm{B})$ and $(\mathrm{G}): \mathrm{x} 40,000$. RGCs, retinal ganglion cells.

Paraptosis may be underestimated. The widespread occurrence of apoptosis and autophagy is readily recognized by numerous specific markers that are coupled to these processes. However, due to a lack of specific markers (19), paraptosis is often concealed by the effects of apoptosis or autophagy $(25,26)$ and thus its occurrence and distribution may be underestimated.

Evidence demonstrates that paraptosis, apoptosis and/or autophagy may occur simultaneously. Several studies have revealed that paraptosis, accompanied by autophagy and apoptosis, was induced by celastrol, a natural compound (25). Our previous investigations demonstrated that the cell death pathway was concentration- and time-dependent and associated with cell type. We identified that honokiol, a constituent of Magnolia officinalis, induced paraptosis at low concentrations. By contrast, at higher concentrations, honokiol induced caspase-dependent apoptosis in leukemic cells, and these two cellular death processes were in sequence and parallel with the increase in honokiol concentration $(26,27)$. These results imply that, in the same cell, more than one death program may be activated simultaneously. Therefore, based on the hypothesis that glaucomatous RGC death involves the process of paraptosis, we also propose that paraptosis and apoptosis and/or autophagy, may occur alone or simultaneously in RGCs.

High levels of ROS are important in RGC death. ROS, including oxygen ions and peroxides, are synthesized during the production of mitochondrial energy, and are highly reactive in signaling pathways for regulating cell growth, differentiation, survival and death. High levels of ROS are important in the signaling of cell death in several neuronal systems, including RGCs (28). Mitochondria may produce enhanced ROS production, that may modulate the autophagy process $(29,30)$. Evidence has revealed that optic nerve crush caused RGCs to produce superoxide anions in response to external oxidative stress, as an early step in signaling apoptosis (31). Furthermore, mitochondrial superoxide was a critical signal in curcumin (a major active component of turmeric)-induced paraptosis in malignant breast cancer cells (32). From the above analysis, we further hypothesize that mitochondria may generate enhanced ROS production, that subsequently contributes to the induction of paraptosis in glaucomatous RGCs.

\section{Preliminary findings of hypothesis}

Our results indicated that swelling mitochondria and vacuoles were detected within 5 and 7 days, following optic nerve crush injury by clamping, in RGCs of adult rats and that these vacuoles originated from the mitochondria. In this investigation, the optic nerve of adult wistar rats underwent crushing as described previously (31). Male wistar rats weighing between 200 and 250 g were purchased from the Laboratory Animal Center, School of Medicine of Xi'an Jiaotong University (Xi'an, Shaanxi, China). All animals received human care in compliance with the ARVO Statement for the Use of Animals in Ophthalmic and Vision Research. The present study was approved by the ethics committee of Shaanxi Institute of Ophthalmology (Xi'an, China). Briefly, the rats were anesthetized and the optic nerves were gently crushed with blunt forceps for $50 \mathrm{sec}$, sparing the retinal and optic disc circulations. The rats were then returned to their cages and sacrificed 
at the time points of day 3,5 or 7 and retinal ultrastructure impairment was analyzed using a transmission electron microscope (H-7650; Hitachi, Tokyo, Japan). All procedures were performed aseptically and on the left eye, with the right eye serving as a sham-operated control. The optic nerve crush injury by clamping induced ultrastructure changes in the RGCs, as demonstrated in Fig. 2.

All the images of RGCs had well-defined plasma membranes and uniformly distributed chromatin in the nucleus, suggesting apoptosis had not evolved in the RGCs within 5 and 7 days following optic nerve crush injury. However, the images in Fig. 2C and 2D demonstrate swollen mitochondria with disintegrated cristae and decreased matrix density. At day 5 and 7, the rate of mitochondrial fusion increased until the cells were almost fully occupied by several large mega mitochondria. However, autophagosomes containing cytoplasmic organelles were not detected, demonstrating that the cell death was not due to autophagy. All the ultrastructural analysis confirmed that the mitochondria were targeted in the RGCs within 5 and 7 days following optic nerve crush injury and the type of RGC death was neither apoptosis nor autophagy, but instead fitted the criteria of paraptosis $(15,18)$.

Glaucoma is a multi-factorial group of eye diseases that result in optic nerve damage, particularly axonal damage, which triggers RGC cell death and the subsequent loss of vision. The optic nerve crush injury rat model is an important experimental disease model for glaucoma and in our study, this model was utilized to examine the injury and morphological changes of RGCs.

Taken together, the above study confirmed that optic nerve crush injury triggered paraptosis in RGCs, suggesting that paraptosis may be involved in glaucomatous RGC death. Further investigations are required to determine whether the excessive production of ROS is triggered in glaucomatous RGCs. However, ultrastructural analysis of swollen mitochondria with disintegrated cristae and intact endoplasmic reticulum further confirmed the hypothesis that mitochondria are important in triggering paraptosis in glaucomatous RGCs.

\section{Conclusion}

In the present review, the evidence suggesting that progressive death of RGCs in glaucoma involves another novel non-apoptotic PCD termed 'paraptosis' in the early stages of glaucoma has been summarized. We conceive this to be a two step process: (i) an excessive production of ROS impairs the mitochondria in glaucomatous RGCs and (ii) the damage to the mitochondria leads to RGC paraptosis. In addition, paraptosis and apoptosis, possibly even together with autophagy, may occur simultaneously in RGCs in the moderate and/or severe stages of glaucoma. Further studies are under way to confirm this theory. These hypotheses stand to enhance the understanding of the mechanisms of glaucomatous RGC damage and provide a novel strategy for protecting RGC by inhibiting paraptosis. Furthermore, we expect this will facilitate the identification of a number of chemical compounds/proteins that directly delay or prevent paraprosis-induced RGC death and thus improve visual damage caused by glaucoma.

\section{Acknowledgements}

This study was supported by the International Cooperation Project of Science and Technology Department of Shaanxi Province (No. 2011 KW-39), Xiamen Science and Technology Project (No. 3502Z20116011, 3502Z20134040) and National Natural Science Foundation of China (No. 81170841).

\section{References}

1. Garcia-Frigola C, Carreres MI, Vegar C and Herrera E: Gene delivery into mouse retinal ganglion cells by in utero electroporation. BMC Dev Biol 7: 103, 2007.

2. Ebneter A, Casson RJ, Wood JP and Chidlow G: Microglial activation in the visual pathway in experimental glaucoma: spatiotemporal characterization and correlation with axonal injury. Invest Ophthalmol Vis Sci 51: 6448-6460, 2010.

3. Leon S, Yin Y, Nguyen J, Irwin N and Benowitz LI: Lens injury stimulates axon regeneration in the mature rat optic nerve. J Neurosci 20: 4615-4626, 2000.

4. Kuehn MH, Fingert JH and Kwon YH. Retinal ganglion cell death in glaucoma: mechanisms and neuroprotective strategies. Ophthalmol Clin North Am 18: 383-395, 2005.

5. Guo L, Moss SE, Alexander RA, Ali RR, Fitzke FW and Cordeiro MF: Retinal ganglion cell apoptosis in glaucoma is related to intraocular pressure and IOP-induced effects on extracellular matrix. Invest Ophthalmol Vis Sci 46: 175-182, 2005.

6. Guo L, Salt TE, Maass A, Luong V, Moss SE, Fitzke FW and Cordeiro MF: Assessment of neuroprotective effects of glutamate modulation on glaucoma-related retinal ganglion cell apoptosis in vivo. Invest Ophthalmol Vis Sci 47: 626-633, 2006.

7. Chen J, Miao Y, Wang XH and Wang Z: Elevation of $\mathrm{p}-\mathrm{NR} 2 \mathrm{~A}(\mathrm{~S} 1232)$ by $\mathrm{Cdk} 5 / \mathrm{p} 35$ contributes to retinal ganglion cell apoptosis in a rat experimental glaucoma model. Neurobiol Dis 43: 455-464, 2011.

8. Tezel G and Yang X: Caspase-independent component of retinal ganglion cell death, in vitro. Invest Ophthalmol Vis Sci 45: 4049-4059, 2004.

9. Spalding KL, Dharmarajan AM and Harvey AR: Caspase-independent retinal ganglion cell death after target ablation in the neonatal rat. Eur J Neurosci 21: 33-45, 2005.

10. Ravindran J, Prasad S and Aggarwal BB. Curcumin and cancer cells: how many ways can curry kill tumor cells selectively? AAPS J 11: 495-510, 2009.

11. Madeo F, Tavernarakis N and Kroemer G: Can autophagy promote longevity? Nat Cell Biol 12: 842-846, 2010.

12. Morselli E, Galluzzi L, Kepp O, Criollo A, Maiuri MC, Tavernarakis N, Madeo F and Kroemer G: Autophagy mediates pharmacological lifespan extension by spermidine and resveratrol. Aging (Albany NY) 1: 961-970, 2009.

13. Rodríguez-Muela N, Germain F, Mariño G, Fitze PS and Boya P: Autophagy promotes survival of retinal ganglion cells after optic nerve axotomy in mice. Cell Death Differ 19: 162-169, 2012.

14. Kim SH, Munemasa Y, Kwong JM, Ahn JH, Mareninov S, Gordon LK, Caprioli J and Piri N: Activation of autophagy in retinal ganglion cells. J Neurosci Res 86: 2943-2951, 2008.

15. Sperandio S, Poksay K, de Belle I, Lafuente MJ, Liu B, Nasir J and Bredesen DE: Paraptosis: mediation by MAP kinases and inhibition by AIP-1/Alix. Cell Death Differ 11: 1066-1075, 2004.

16. Bröker LE, Kruyt FA and Giaccone G: Cell death independent of caspases: a review. Clin Cancer Res 11: 3155-3162, 2005.

17. Kang R, Zeh HJ, Lotze MT and Tang D: The Beclin 1 network regulates autophagy and apoptosis. Cell Death Differ 18: 571-580, 2011.

18. Sperandio S, de Belle I and Bredesen DE: An alternative, nonapoptotic form of programmed cell death. Proc Natl Acad Sci USA 97: 14376-14381, 2000.

19. Sperandio S, Poksay KS, Schilling B, Crippen D, Gibson BW and Bredesen DE: Identification of new modulators and protein alterations in non-apoptotic programmed cell death. J Cell Biochem 111: 1401-1412, 2010.

20. Yang Z and Klionsky DJ: Mammalian autophagy: core molecular machinery and signaling regulation. Curr Opin Cell Biol 22: 124-131, 2010. 
21. Valamanesh F, Torriglia A, Savoldelli M, Gandolphe C, Jeanny JC, BenEzra D and Behar-Cohen F: Glucocorticoids induce retinal toxicity through mechanisms mainly associated with paraptosis. Mol Vis 13: 1746-1757, 2007.

22. Chen Y, Douglass T, Jeffes EW, Xu Q, Williams CC, Arpajirakul N, Delgado C, Kleinman M, Sanchez R, Dan Q, et al: Living T9 glioma cells expressing membrane macrophage colony-stimulating factor produce immediate tumor destruction by polymorphonuclear leukocytes and macrophages via a 'paraptosis'-induced pathway that promotes systemic immunity against intracranial T9 gliomas. Blood 100: 1373-1380, 2002.

23. Jadus MR, Chen Y, Boldaji MT, Delgado C, Sanchez R, Douglass T, Al-Atar U, Schulz W, Lloyd C and Wepsic HT: Human U251MG glioma cells expressing the membrane form of macrophage colony-stimulating factor (mM-CSF) are killed by human monocytes in vitro and are rejected within immunodeficient mice via paraptosis that is associated with increased expression of three different heat shock proteins. Cancer Gene Ther 10: 411-420, 2003.

24. Schneider D, Gerhardt E, Bock J, Müller MM, Wolburg H, Lang F and Schulz JB: Intracellular acidification by inhibition of the $\mathrm{Na}+/ \mathrm{H}+-$ exchanger leads to caspase-independent death of cerebellar granule neurons resembling paraptosis. Cell Death Differ 11: 760-770, 2004.

25. Wang WB, Feng LX, Yue QX, Wu WY, Guan SH, Jiang BH, Yang M, Liu X and Guo DA: Paraptosis accompanied by autophagy and apoptosis was induced by celastrol, a natural compound with influence on proteasome, ER stress and Hsp90. J Cell Physiol 227: 2196-2206, 2010.
26. Wang Y, Yang Z and Zhao X: Honokiol induces paraptosis and apoptosis and exhibits schedule-dependent synergy in combination with imatinib in human leukemia cells. Toxicol Mech Methods 20: 234-241, 2010

27. Wang Y, Zhu X, Yang Z and Zhao X: Honokiol induces caspaseindependent paraptosis via reactive oxygen species production that is accompanied by apoptosis in leukemia cells. Biochem Biophys Res Commun 430: 876-882, 2013.

28. Geiger LK, Kortuem KR, Alexejun C and Levin LA: Reduced redox state allows prolonged survival of axotomized neonatal retinal ganglion cells. Neuroscience 109: 635-642, 2002.

29. Ravikumar B, Sarkar S, Davies JE, Futter M, Garcia-Arencibia M, Green-Thompson ZW, Jimenez-Sanchez M, Korolchuk VI, Lichtenberg M, Luo S, et al: Regulation of mammalian autophagy in physiology and pathophysiology. Physiol Rev 90: 1383-1435, 2010.

30. Li ZY, Yang Y, Ming M and Liu B: Mitochondrial ROS generation for regulation of autophagic pathways in cancer. Biochem Biophys Res Commun 414: 5-8, 2011.

31. Lieven CJ, Hoegger MJ, Schlieve CR and Levin LA: Retinal ganglion cell axotomy induces an increase in intracellular superoxide anion. Invest Ophthalmol Vis Sci 47: 1477-1485, 2006.

32. Yoon MJ, Kim EH, Lim JH, Kwon TK and Choi KS: Superoxide anion and proteasomal dysfunction contribute to curcumin-induced paraptosis of malignant breast cancer cells. Free Radic Biol Med 48: 713-726, 2010. 